\title{
Modelling Resilience in Interdependent Infrastructures: Real-World Considerations
}

\author{
Mong Soon Sim and Kah Wah Lai
}

\begin{abstract}
This presentation discusses the issues that a practitioner may encounter in modelling resilience of a network of interdependent infrastructures. Resilience here focuses on the response and recovery of the infrastructure systems, in the event of disruption. On the model scope, it is not unusual that infrastructures with different operating characteristics have to be taken into account. This is compounded with the challenges to obtain and maintain data for a large network, decipher the specific questions that the user wants to address in the study. On the modelling methodology, traditional OR models are not sufficient to describe the real world applications, as they often do not account for additional requirements. One such requirement is the inclusion of response mechanism of the infrastructures when they encounter disruption at supply or demand side. This presentation will specifically describe eight response mechanisms modelled in a recent study and how they may be incorporated into the traditional OR models as linear constraints via 0-1 indicator and artificial variables. On the implementation, one critical step is to bridge the gap between the modeler and the stakeholder. In order for the model to be used in the users' organization, one strategy is to integrate the model into the user's current Concept of Operations (CONOPS). To do this, the modeler has to look beyond the modeling requirements. One aspect is the model capability to handle an expanding problem size and changing operating environment of the infrastructures. Nowadays, most commercial optimization software allow separation of the data file from the model file; this feature will facilitate this scalability aspect. Lastly, we note that, the key concern is usually not the ability of the optimization algorithm to solve the problem, as commercial optimizers work well for most real-world applications. The difficult issue faced is usually getting the users to use the model well.
\end{abstract}

M.S. Sim $(\varangle) \cdot$ K.W. Lai

DSO National Laboratories, 20 Science Park Drive, Singapore 118230, Singapore

e-mail: smongsoo@dso.org.sg

K.W. Lai

e-mail: 1kahwah@dso.org.sg

(C) Springer International Publishing Switzerland 2016

M.-A. Cardin et al. (eds.), Complex Systems Design \& Management Asia,

Advances in Intelligent Systems and Computing 426,

DOI 10.1007/978-3-319-29643-2_20 